\title{
Investigation of Anatomical Thalamo-Cortical Connectivity and fMRI Activation in Schizophrenia
}

\author{
Stefano Marenco*, 1,4 Jason L Stein 1,4, Antonina A Savostyanova', Fabio Sambataro², Hao-Yang Tan', \\ Aaron L Goldman', Beth A Verchinski', Alan S Barnett', Dwight Dickinson', José A Apud', \\ Joseph H Callicott', Andreas Meyer-Lindenberg ${ }^{3}$ and Daniel R Weinberger' \\ 'Clinical Brain Disorders Branch, Genes, Cognition, and Psychosis Program, National Institute of Mental Health Intramural Research Program, \\ Bethesda, MD, USA; ${ }^{2}$ Brain Center for Motor and Social Cognition, Italian Institute of Technology, Parma, Italy; ${ }^{3}$ Department of Psychiatry and \\ Psychotherapy, Central Institute of Mental Health, Mannheim, Germany
}

\begin{abstract}
The purpose of this study was to examine measures of anatomical connectivity between the thalamus and lateral prefrontal cortex (LPFC) in schizophrenia and to assess their functional implications. We measured thalamocortical connectivity with diffusion tensor imaging (DTI) and probabilistic tractography in 15 patients with schizophrenia and 22 age- and sex-matched controls. The relationship between thalamocortical connectivity and prefrontal cortical blood-oxygenation-level-dependent (BOLD) functional activity as well as behavioral performance during working memory was examined in a subsample of 9 patients and 18 controls. Compared with controls, schizophrenia patients showed reduced total connectivity of the thalamus to only one of six cortical regions, the LPFC. The size of the thalamic region with at least $25 \%$ of model fibers reaching the LPFC was also reduced in patients compared with controls. The total thalamocortical connectivity to the LPFC predicted working memory task performance and also correlated with LPFC BOLD activation. Notably, the correlation with BOLD activation was accentuated in patients as compared with controls in the ventral LPFC. These results suggest that thalamocortical connectivity to the LPFC is altered in schizophrenia with functional consequences on working memory processing in LPFC.

Neuropsychopharmacology (2012) 37, 499-507; doi:I0.1038/npp.201।.215; published online 28 September 20। I
\end{abstract}

Keywords: diffusion tensor imaging (DTI), probabilistic tractography; medio-dorsal nucleus; prefrontal cortex; working memory; reproducibility

\section{INTRODUCTION}

The thalamus features prominently in major hypotheses about the neural circuitry implicated in schizophrenia (Andreasen et al, 1996), and evidence for its involvement derives from multiple fields of investigation but remains controversial (reviewed in Byne et al, 2009). Abnormalities in the medio-dorsal, pulvinar, and centromedian nuclei of the thalamus, all connected to the lateral prefrontal cortex (LPFC), a site of well-documented abnormalities in schizophrenia (Weinberger et al, 2001), have been reported with several methods (eg, Andrews et al, 2006; Byne et al, 2002; Byne et al, 2007; Harms et al, 2007; Hazlett et al, 1999; Kemether et al, 2003; Kessler et al, 2009; Kumari et al, 2010;

\footnotetext{
*Correspondence: Dr S Marenco, Clinical Brain Disorders Branch, Genes, Cognition, and Psychosis Program, National Institute of Mental Health Intramural research program, National Institute of Mental Health, 10 Center Drive, Building 10, room 4S235, Bethesda, MD 20892, USA, Tel: + I 30 I 435 8964, Fax: + I 30 I 4807795 ,

E-mail: marencos@mail.nih.gov

${ }^{4}$ These authors contributed equally to this paper

Received 28 January 20 I I; revised I August 201।; accepted I8 August 2011
}

Lehrer et al, 2005; Popken et al, 2000; Rose et al, 2006, but see, for example, Danos et al, 2005; Dorph-Petersen et al, 2004; Kreczmanski et al, 2007 for negative findings). Some functional MRI (fMRI) studies found abnormalities in the correlation between the activity in the thalamus and the PFC in schizophrenia (eg, Meda et al, 2010; Schlosser et al, 2003; Welsh et al, 2010; Zhou et al, 2007), which may reflect alterations in anatomical connectivity. Thus, the changes in the coordinated functioning of the PFC and the thalamus might be relevant to the pathophysiology of schizophrenia.

Whether there is an abnormality in anatomical connectivity between the thalamus and the prefrontal cortex in schizophrenia is an important knowledge gap that might be addressed with the latest advances in probabilistic tractography via diffusion tensor imaging (DTI). This technique has allowed researchers to segment the thalamus in functionally relevant connectivity-defined regions (CDRs) based on their preferential connectivity to particular cortical areas (Behrens et al, 2003a, b; Johansen-Berg et al, 2005; Klein et al, 2010). Here, we used this methodology to test the following hypotheses: (1) that the degree of connectivity from the thalamus to the LPFC defined via probabilistic tractography is reduced in schizophrenia, 
(2) that thalamocortical connectivity would predict performance during a working memory task and (3) associated prefrontal blood-oxygenation-level-dependent (BOLD) activation during fMRI. Both working memory performance and prefrontal activation have been found to index cortical dysfunction and to be related to genetic risk for schizophrenia (Callicott et al, 2003a; Egan et al, 2001; MeyerLindenberg and Weinberger, 2006).

\section{PATIENTS AND METHODS}

In all, 19 patients with schizophrenia and 26 healthy volunteers participated in the study. Participants were assessed as detailed in (Egan et al, 2001). Exclusions based on the history of other axis I disorders (including substance abuse), significant medical history, or excessive movement in the scanner (corrupting a substantial portion of the acquired images) resulted in the final group of 15 patients and 22 controls reported here. All participants had taken part in the Clinical Brain Disorders Branch 'Sibling Study' (NCT00001486). Five patients with schizophrenia were recruited as outpatients, whereas the rest were recruited from our inpatient ward (NCT00001247). No T2 hyperintensities were observed on the MRI scans of the participants. At the time of study, all patients were treated with second-generation antipsychotics and adjunctive medications.

DTI sessions were conducted using a GE 1.5T Signa scanner (GE, Milwaukee, WI) with an axial single shot echo planar imaging (EPI) sequence (TE $83.7 \mathrm{~ms}, 80$ slices with isotropic voxel size $2 \times 2 \times 2 \mathrm{~mm}, \mathrm{NEX}=1,80 \times 110$ matrix, field of view: $22 \mathrm{~cm}$ ) with cardiac gating of each individual slice (TR $>10000 \mathrm{~ms}$ ). In all, 107 volumes were acquired for each participant $\left(b_{\max }=1200 \mathrm{~s} / \mathrm{mm}^{2}\right.$; see Supplementary Table S1), with 42 directions acquired with $b$ values $>850 \mathrm{~s} /$ $\mathrm{mm}^{2}$. The directions of diffusion-weighted scans were distributed uniformly in space for each $b$ value acquired. In order to assess the reproducibility of our measures, ten controls repeated one DTI session twice, with an average time between scans of $61 \pm 32$ (SD) days.

In separate sessions, three-dimensional structural MRI and fMRI scans were acquired. A $T_{1}$-weighted spoiled gradient $(\mathrm{SPGR})$ sequence $(\mathrm{TR} / \mathrm{TE}=2400 / 5 \mathrm{~ms}$, flip angle $=$ $45^{\circ}$, with 124 sagittal slices, $256 \times 256$ matrix, FOV $24 \mathrm{~cm}$, $0.94 \times 0.94 \times 1.5 \mathrm{~mm}^{3}$ voxel size) at $1.5 \mathrm{~T}(\mathrm{GE})$ was used for the structural acquisition whereas a $3 \mathrm{~T}$ system (GE) was used for fMRI EPI acquisition with TR/TE $=2000 / 30 \mathrm{~ms}$, flip angle $=90^{\circ}$, FOV $24 \mathrm{~cm}, 64 \times 64$ matrix, $3.75 \times 3.75 \times 6 \mathrm{~mm}$ voxel size, 24 contiguous slices. The task paradigm used during fMRI was the 2-back working memory task (30 epochs of 0 -back alternating with 2-back as previously described in Callicott et al (2003b)).

The Freesurfer software package (http://surfer.nmr.mgh. harvard.edu) was used to generate eight bilateral cortical (Figure 1) and one thalamic region of interest (ROIs) from the $T_{1}$-weighted structural images. ROIs were then transformed into DTI space for tractography. Affine registration (Jenkinson and Smith, 2001) using a normalized mutual information cost function with 12 degrees of freedom was used for each participant to derive transformation matrices from the non-diffusion-weighted image of the DTI series to

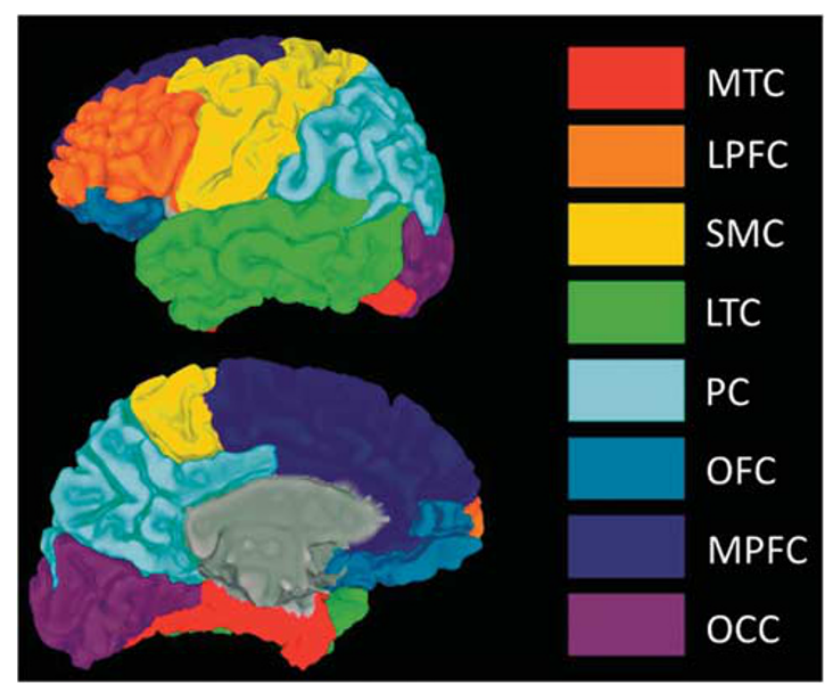

Figure I Cortical ROls in one healthy volunteer. The subdivisions of the cortex used in the analysis are shown for the left hemisphere in a lateral (top) and medial (bottom) view with a color bar for reference. Bilateral ROls were used in the analysis, whereas unilateral ROls are shown here for visualization. The following original Freesurfer parcellations (Desikan et al, 2006) were combined to form the larger ROls used here: orbitofrontal cortex (OFC: pars orbitalis, medial orbitofrontal cortex, lateral orbitofrontal cortex), medial prefrontal cortex (MPFC: caudal anterior cingulate, rostral anterior cingulate, superior frontal gyrus), lateral prefrontal cortex (LPFC: pars triangularis, frontal pole, rostral middle frontal gyrus, pars opercularis), sensorimotor cortex (SMC: precentral gyrus, caudal middle frontal gyrus, post-central gyrus, paracentral lobule), parietal cortex (PC: inferior parietal cortex, supramarginal gyrus, precuneus cortex, posterior cingulate cortex, isthmus cingulate, superior parietal cortex), medial temporal cortex (MTC: entorhinal cortex, parahippocampal gyrus, fusiform gyrus), lateral temporal cortex (LTC: transverse temporal cortex, superior temporal gyrus, banks of the superior temporal sulcus, inferior temporal gyrus, middle temporal gyrus, temporal pole), and occipital cortex (OCC: pericalcarine cortex, lingual gyrus, lateral occipital cortex, cuneus cortex). Graphics were created using SUMA, part of the AFNI package (http://afni.nimh.nih.gov/afni/suma).

the structural $T_{1}$-weighted image. The inverse of those transformation matrices was then applied to convert the binary cortical ROIs to diffusion space with trilinear interpolation. Cortical ROIs in diffusion space were thresholded at 0.3 , re-binarized, and corrected such that there were no overlapping voxels among regions. To ensure that no white matter voxels were included in the cortical ROIs, all voxels with fractional anisotropy $>0.2$ were excluded from the cortical ROIs. Bilateral thalamic ROIs in diffusion space were thresholded at 0.6 and re-binarized. These thresholds were established after careful review of overlayed masks and registered $T_{1}$ and non-diffusionweighted images. We confirmed visually in each individual that the thalamic mask did not grossly exceed the borders of the thalamus after registration, while encompassing the entire $T_{1}$-weighted visible structure (the borders of the thalamus are not easily detectable in the non-diffusionweighted image). For the cortex, a more liberal threshold for binarization was chosen to retain cortical voxels while preventing the extension of the mask into white matter.

All tools for DTI processing belonged to the FMRIB Diffusion Toolbox in the FMRIB Software Library (FSL 4.0; http://www.fmrib.ox.ac.uk/fsl/). The DTI series was first manually reviewed to eliminate artifacts. Volumes where 
sudden motion had occurred (resulting in slices with low signal) were eliminated. We believe that the rejection of these volumes did not influence our results because all participants had at least seven volumes with $b$ values $<10 \mathrm{~s} /$ $\mathrm{mm}^{2}$ and 42 directions with $b>850 \mathrm{~s} / \mathrm{mm}^{2}$. Images were corrected for distortion caused by eddy currents and head motion using an affine registration to the first nondiffusion-weighted volume (Jenkinson and Smith, 2001).

The brain was extracted and the diffusion parameters were estimated (Behrens et al, 2007), giving a probability distribution function of two fibers in each voxel of the brain. For each voxel in the thalamic mask, 5000 samples were sent through the connectivity distribution using probabilistic tractography. Probabilistic tractography was run bilaterally as pilot studies showed that reproducibility was somewhat improved over unilateral measures.

Total percent connectivity was calculated as the number of samples from any thalamic voxel reaching the corresponding cortical ROI divided by the total number of samples from all thalamic voxels reaching any cortical ROI. This is a measure of total tractography-defined connectivity from the thalamus to a particular cortical area, independent of where the tract originated from inside the thalamus.

When $>25 \%$ of the total number of samples originating in a thalamic voxel reached a cortical ROI, that voxel was assigned to a CDR, considered to be connected to the homonymous cortical ROI. The total number of voxels belonging to a CDR divided by the total number of voxels in the thalamus of that individual was the outcome measure. This is a measure of the relative size of the thalamic CDR connected via tractography to a particular cortical area.

The two dependent variables for each cortical ROI were used as within effects in separate analyses of variance, where diagnostic group was the between effect. Two ROIs (medial temporal and orbito-frontal cortices) were dropped from this analysis due to poor reproducibility across two repeated scans of 10 normal controls (Supplementary Methods $a$ ). In these analyses, main effects of the region were of no interest, and will not be reported further, although we focused on main effects of diagnosis or ROI-bydiagnosis interactions. If the latter was significant, post-hoc $t$-tests were run for each ROI. These were not corrected for multiple comparisons as the overall significance protects from type I error. The effect of possible confounding variables on LPFC percent total connections was assessed as detailed in the Supplementary Methods $b$. All statistics of this kind were run with Statistica release 7 (StatSoft, Tulsa, OK).

fMRI was analyzed for a subset of 27 individuals (18 healthy subjects and 9 patients with schizophrenia: Supplementary Methods $c$, Table S3) during an n-back working memory task. All fMRI data were preprocessed and analyzed with SPM5 software (http://www.fil.ion.ucl.ac.uk/ $\mathrm{spm}$ ). All fMRI data sets met the criteria for quality control as described previously (Callicott et al, 2003b). All fMRI data were pre-processed and spatially normalized to the common stereotactic space provided by the Montreal Neurologic Institute (MNI) and analyzed using a general linear model. For each experimental condition, a box car model convolved with the hemodynamic response function at each voxel was modeled. Linear contrasts were computed producing voxel-wise $\mathrm{t}$-statistical parameter maps for 2-back relative to 0-back and entered into second-level analyses.

Two second-level multiple regression models were tested on the contrast images (2-back-0-back), with the subject as a random factor: one to detect voxels with a significant relationship to LPFC connectivity where both groups were assumed to have equal slopes; the other to detect voxels with significantly different slopes between the diagnostic groups. The first model had LPFC connectivity, diagnosis, age, sex, and 2-back performance as covariates. The second model was constructed including the following predictors: two diagnostic group predictors, two LPFC connectivity predictors (one per diagnostic group), age, sex, and n-back accuracy. The contrast between the two LPFC connectivity vectors (adjusted by group means, age, sex, and n-back performance within each group) was then used to assess group differences in the slope of the regression.

Levels of significance to detect associations between LPFC connectivity and BOLD activation were set at $p=0.005$ uncorrected for multiple comparisons. Results are reported in a ROI constructed by normalizing to the MNI template and then averaging the bilateral LPFC ROIs used as tractography targets. The resulting image was smoothed with a $3 \times 3 \times 3 \mathrm{~mm}$ FWHM filter mimicking the fMRI resolution and then thresholded at a value of 0.4 for binarization. Corrections for multiple comparisons were performed at the cluster extent level, using the cluster size threshold computed via AlphaSim, a function available in AFNI (http://afni.nimh.nih.gov/). This program was run for each individual with 1000 iterations, within the ROI described above, and a cluster connectivity radius of $3.1 \mathrm{~mm}$. AlphaSim estimated the rate of false positive voxels within a cluster that are expected given a threshold of $p<0.005$ uncorrected. For a specific $\alpha$, any cluster size larger than this number can be considered statistically significant after correction for multiple comparisons. For any group analysis, the critical cluster size is calculated averaging this measure across all individuals. In our analysis, for $\alpha=0.05$ the average cluster extent threshold was $621 \mu \mathrm{l}$ ( 23 voxels).

The relationship between LPFC total percent connectivity and number of correct responses on the 2-back was assessed with Pearson's $r$.

\section{RESULTS}

The patients $(n=15)$ and controls $(n=22)$ did not significantly differ in age, sex, or handedness (Table 1). Overall quality of the tractography results was assessed in several ways, as detailed in the Supplementary Results (Figures S1, S2, S3, S4), and appeared to be consistent with known anatomy.

A significant diagnosis-by-ROI interaction was found for percent total connectivity $\left(F_{5,175}=22.4, p=0.03\right)$ and for CDR size $\left(F_{5,175}=2.38, p=0.04\right.$ : Figures 2 and 3 , and Supplementary Table S4). Patients with schizophrenia had on average lower thalamocortical connectivity and CDR size than controls in the LPFC, whereas they had elevated total connectivity to the somato-motor cortex.

There was no correlation of measures of connectivity with age, years of education or Wide Range Achievement Test in 
Table I Subject Characteristics

\begin{tabular}{|c|c|c|c|}
\hline & $\begin{array}{l}\text { Patients with } \\
\text { schizophrenia } \\
\quad(n=15)\end{array}$ & $\begin{array}{c}\text { Healthy } \\
\text { volunteers } \\
(n=22)\end{array}$ & $\begin{array}{l}\text { Statistical } \\
\text { test }\end{array}$ \\
\hline Age, years (mean $\pm S D)$ & $30.6 \pm 12.5$ & $34.1 \pm 12.0$ & $\begin{aligned} t_{35} & =-0.85 \\
p & =0.40\end{aligned}$ \\
\hline Gender & $11 \mathrm{M}, 4 \mathrm{~F}$ & $17 \mathrm{M}, 5 \mathrm{~F}$ & $\begin{array}{c}\chi^{2}=0.08 \\
p=0.78\end{array}$ \\
\hline Handedness $^{\mathrm{a}}$ & $12 \mathrm{R}, 2 \mathrm{~L}$ & $18 \mathrm{R}, 4 \mathrm{~L}$ & $\begin{aligned} \chi^{2} & =0.0935, \\
p & =0.76\end{aligned}$ \\
\hline Family SES ${ }^{\mathrm{a}}$ & $46.5 \pm 14$ & $52 \pm \mid 1.1$ & $\begin{aligned} t_{34} & =-1.32 \\
p & =0.2\end{aligned}$ \\
\hline \multicolumn{4}{|l|}{ Cognitive measures } \\
\hline Years of education & $13.5 \pm 1.9$ & $15.5 \pm 1.9$ & $\begin{aligned} t_{35} & =-3.10 \\
p & <0.01\end{aligned}$ \\
\hline WRAT reading score & $98.9 \pm 14.9$ & $109.4 \pm 9.29$ & $\begin{aligned} t_{35} & =-2.63 \\
p & =0.01\end{aligned}$ \\
\hline \multicolumn{4}{|l|}{ Clinical measures } \\
\hline Age of onset, years & $19.0 \pm 3.0$ & & \\
\hline Duration of illness (years) ${ }^{b}$ & $14.1 \pm 12.0$ & & \\
\hline Chlorpromazine equiv c,a & $595.8 \pm 491.1$ & & \\
\hline PANSS positive factor ${ }^{d}$ & $12.9 \pm 4.0$ & & \\
\hline PANSS negative factor ${ }^{d}$ & $15.4 \pm 4.0$ & & \\
\hline PANSS cognitive factor ${ }^{d}$ & $|4| \pm 3.5$. & & \\
\hline PANSS excitement factor ${ }^{d}$ & $9.0 \pm 1.8$ & & \\
\hline PANSS depression factor ${ }^{d}$ & $9.0 \pm 3.1$ & & \\
\hline
\end{tabular}

Abreviations: F, female; L, left-handed; M, male; PANSS, Positive and Negative Syndrome Scale; R, right-handed; WRAT, Wide Range Achievement Test.

${ }^{a}$ Data missing for one patient. SES based on Hollingshead (1975).

bBased on the age at first hospitalization.

${ }^{c}$ Calculated using Woods (2003).

'Patients' symptoms were rated with the PANSS by an experienced nurse or a psychiatrist and were summarized in five factor scores (Lindenmayer et al, 1995).

the whole group, or with chlorpromazine equivalents and duration of illness in the patient group. There were no significant differences between groups in total thalamic or frontal cortical volumes (Supplementary Table S5: this was true both for absolute volumetric measures and for those normalized to intra-cranial volume), or in the average tract properties connecting the thalamic CDR and the LPFC (Supplementary Table S6).

In the fMRI study of working memory, patients and controls had similar performance in terms of accuracy and reaction time $(p>0.15)$ and performance was used as a covariate in the analysis. Thalamic LPFC percent total connectivity correlated with cortical activation in the LPFC bilaterally (Figure 4a, maximum effect: $r^{2}=0.33, p=0.0017$ uncorrected, cluster size $648 \mu \mathrm{l}$ ) for the model with equal slopes across groups, although patients with schizophrenia had steeper slopes than controls in the right ventral LPFC (Figure $4 \mathrm{~b}, p=0.0007$ uncorrected, cluster size $648 \mu \mathrm{l}$ ). Both relationships occurred in clusters larger than $621 \mu \mathrm{l}$, thus corrected for multiple comparisons. LPFC total percent connectivity was significantly correlated with 2-back performance in the whole group (Figure $4 c, r^{2}=0.19$, $p=0.02)$.
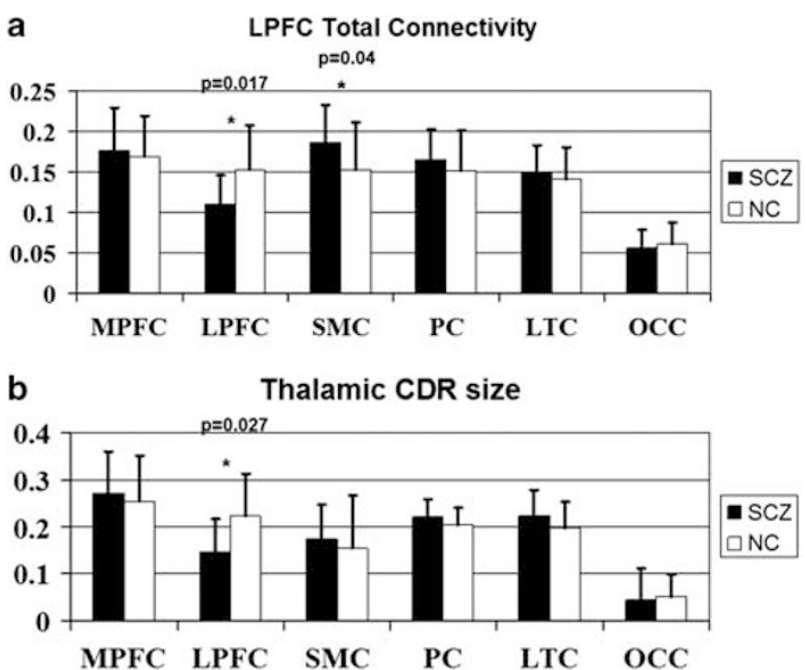

Figure 2 Results for total percent connectivity and CDR size. (a) Total percent connectivity for the six cortical ROls included in the analysis. (b) Thalamic CDR size for the six regions included in the analysis. $P$ values represent post-hoc unpaired $t$-tests. ${ }^{*} P<0.05$ on the post-hoc tests. Error bars represent SD. LTC, lateral temporal cortex; LPFC, lateral PFC; MPFC, medial prefrontal cortex; OCC, occipital cortex; PC, parietal cortex; SMC, somato-motor cortex.
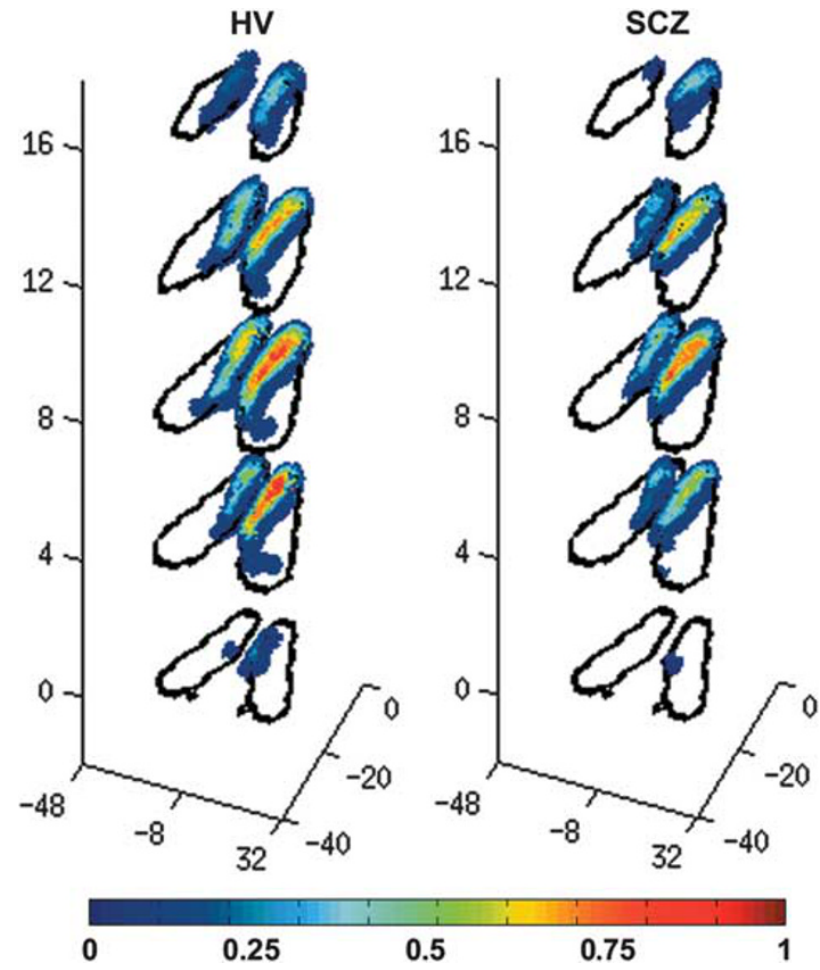

Figure 3 Comparison of the spatial consistency of LPFC thalamic CDRs between patients with schizophrenia and controls. Binary masks of the thalamic CDRs for the healthy volunteers $(n=22)$ and patients with schizophrenia $(n=15)$ were normalized to a standard template (Wake Forest University Pickatlas: http://fmri.wfubmc.edu/cms/softwarePickAtlas), averaged, and thresholded to show only those voxels with $>10 \%$ of the subjects represented at that location. The black border outlines five slices of the thalamus, oriented from top to bottom, with the axes showing the $\mathrm{mm}$ coordinates in standard MNI space. Warmer colors illustrate a higher spatial consistency across subjects, with dark red representing 100\% of subjects present at a particular location. Graphics were created using Matlab (Mathworks, Natick, MA). 
a
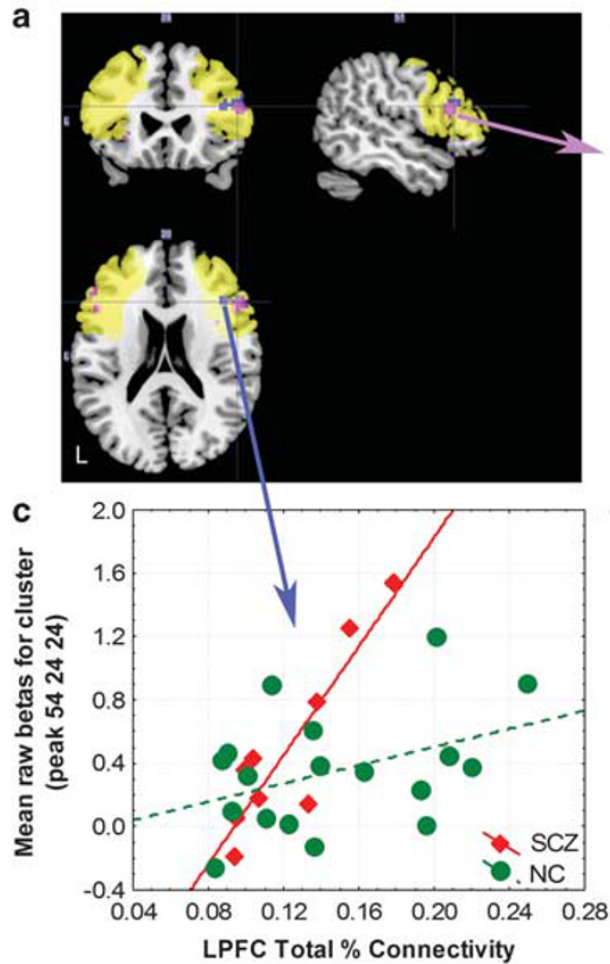

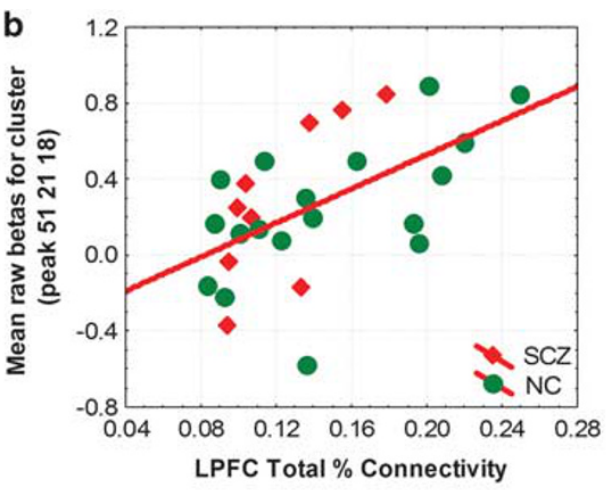

d

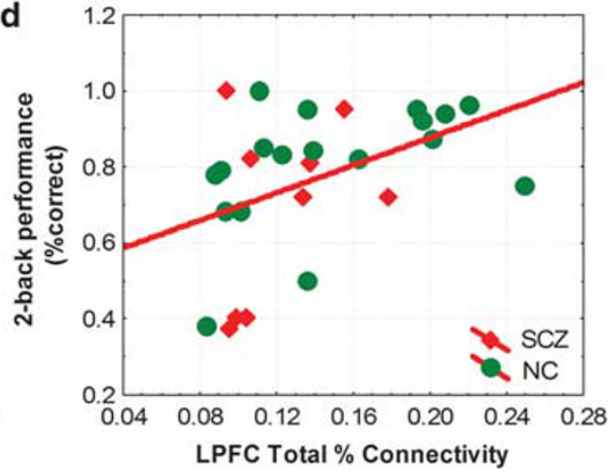

Figure 4 Correlation of prefrontal BOLD activation and LPFC total percent connectivity. (a) The significant clusters are shown superimposed on the MNI single subject template. The yellow area represents the region of interest used to constrain the analysis, the pink area corresponds to the cluster (maximum at voxel coordinates 5 I 21 18) where both groups had similar positive slopes for the correlation between LPFC total percent connectivity and BOLD, whereas the blue area corresponds to the cluster (maximum at voxel 5424 24) where slopes differed significantly across groups. (b) Correlation between LPFC total percent connectivity and BOLD parameter estimates for the cluster with the peak at voxel 5121 I 8 (raw $\beta$ values adjusted for the session mean of the individual subject, $n=27, r=0.56, p=0.0027, z$ for peak voxel $=3.33$ ). The cluster surrounding the peak of significance encompassed 24 voxels $(648 \mu \mathrm{l})$. This exceeded the threshold of 23 voxels $(62 \mathrm{l} \mu \mathrm{l})$ found by AlphaSim and can therefore be considered corrected for multiple comparisons. (c) Significant difference in the correlation between LPFC connectivity and BOLD activation in the cluster surrounding voxel 542424 (raw $\beta$ values adjusted for the session mean, normal controls (NC): $n=18, r=0.4$, patients with schizophrenia (SCZ): $n=9, r=0.9,24$ voxel extent or $648 \mu l, z$ for peak voxel $=3.38$, $p=0.0007$, uncorrected). (d) Correlation between LPFC connectivity and performance on the 2-back working memory task ( $r=0.43$, $p=0.02)$ for the whole group. Correlation coefficients for patients and controls did not differ from each other (SCZ $=0.38, N O R=0.44$, Fischer's transformation: $z=-0.15$, $p=0.88)$

Given these results, we also investigated the relationship between percent total connectivity from the thalamus to two other cortical masks where significant BOLD activation occurs during the n-back task: parietal and medial prefrontal cortices (Supplementary Methods $d$, Supplementary Results, and Supplementary Figures S5 and S6).

\section{DISCUSSION}

Based on suggestive but controversial findings from postmortem studies and on data acquired with structural and functional neuroimaging, we had hypothesized that measures indexing thalamocortical connectivity to the LPFC would be reduced in patients with schizophrenia as compared with normal controls. Probabilistic tractography data obtained in vivo support the central hypothesis of this study. The measures derived with this method were highly reproducible in two repeated scans of a subset of the control subjects (ICC >0.82); the effect size of the differences between diagnostic groups appeared robust (Cohen's $d \sim 0.8$ ), and unlikely to be due to several possible confounding factors (eg, age and chlorpromazine equivalents). Our data offer a possible anatomical substrate for differences in functional connectivity between patients with schizophrenia and controls previously described with fMRI (Meda et al, 2010; Schlosser et al, 2003; Welsh et al, 2010; Zhou et al, 2007) and are consistent with a reduction in connectivity from multiple sources in the thalamus to the LPFC. Besides three studies showing that neurons specifically projecting to LPFC in the medio-dorsal nucleus of the thalamus may be reduced in schizophrenia (Byne et al, 2002; Popken et al, 2000; Young et al, 2000), reduced sizes of the pulvinar, of the centromedian nuclei (both projecting in part to the LPFC: Byne et al, 2007; Kemether et al, 2003) and reductions in oligodendrocyte number in the anterior principal nucleus (Byne et al, 2006, 2008) were found in schizophrenia. An unexpected increase in total connectivity from the thalamus to the sensorimotor cortices was found, but it is difficult to interpret this in the absence of a prior hypothesis. One possibility is that this is an artifact of the relative measures used here to assess thalamic connectivity: a decrease in connectivity to the LPFC necessarily has to correspond to a decrease somewhere else, however, it is unclear why this would be more prominent in the sensorimotor cortices rather than distributed in a more diffuse fashion. 
It should be stressed that the reproducibility over repeated DTI sessions of the thalamo-LPFC connectivity measures reported here was satisfactory, with ICCs $>0.69$, a result consistent with another recent investigation where spatial reproducibility of this method was studied in detail (Traynor et al, 2010). The poor reproducibility in the OFC and MTC (Supplementary Table S2) were most likely due to EPI distortions for the orbitofrontal regions and the presence of complex fiber architecture on the pathway from the thalamus to the medial temporal cortex that is not fully accounted for by the current probabilistic connectivity models.

This is not the first study pointing to altered diffusion parameters in the thalamus or thalamocortical pathways. DTI findings supporting altered integrity of thalamocortical connectivity in schizophrenia include controversial alterations of fractional anisotropy in the anterior limb of the internal capsule (ALIC) (Buchsbaum et al, 2006; Kubicki et al, 2005; Mamah et al, 2010; Sprooten et al, 2009; Sussmann et al, 2009; Zhang et al, 2010; Zhou et al, 2008, but see Ashtari et al, 2007; Kito et al, 2009; Kyriakopoulos et al, 2008; Szeszko et al, 2005; White et al, 2007 for negative findings in younger individuals and Beasley et al, 2009 for lack of evidence of myelin abnormalities post-mortem), of the tracts connecting to the LPFC (Kim et al, 2008; Oh et al, 2009) and altered FA (Hashimoto et al, 2009) or mean diffusivity in the thalamus itself (Agarwal et al, 2008; Rose et al, 2006; Spoletini et al, 2011).

Kim et al (2007) have performed a similar DTI study, with an identical $a$-priori hypothesis, but found only differences in the thalamic CDR defined by connectivity to the orbitofrontal, parietal, and medial prefrontal cortices. No measure of reproducibility was reported in that paper and several methodological issues might have prevented those authors from finding similar results to ours: the more limited number of directions used, maximal $b$ values of $600 \mathrm{~mm}^{2} / \mathrm{s}$ (which will limit the accuracy of the determination of the direction of diffusion), the use of a larger definition for the LPFC ROI that included cortical structures not necessarily altered in schizophrenia, and the use of a processing software limited to modeling one fiber per voxel.

It is possible that these alterations in thalamocortical connectivity were due to alterations in diffusion properties in the tract connecting the thalamus to the LPFC, as detailed above. In this small sample, we could not detect a difference in the average FA or mean diffusivity for the tract connecting the thalamus to the LPFC (Supplementary Table S6), however, more subtle local differences in diffusion properties may exist along the tract that were not detected in the analysis of average values (Oh et al, 2009).

Although the weight of the evidence points towards a reduction in thalamic volume in schizophrenia (for metaanalyses see Adriano et al, 2011; Konick and Friedman, 2001, and for a recent large study see Rimol et al, 2010), it is possible that we did not detect significant differences in thalamic or cortical volumes because of limited power due to the sample size. Our findings, however, are consistent with those collected in a much larger cohort of patients and controls acquired and processed with identical methodology (Goldman et al, 2008). These negative findings are of interest because they make it more likely that the main results of this study are primary and not secondary to volumetric changes or generic alterations in white matter integrity in the whole thalamus or in the ALIC and anterior thalamic radiations mentioned above. At the very least, though, in this small sample tractography-derived measures were more sensitive than volumetric measures in detecting anatomical differences in schizophrenia.

Using a multimodal approach, we also investigated whether the observed thalamic connectivity measures were biologically relevant for the activity of the LPFC during the performance of working memory. Indeed, percent total connectivity to the LPFC predicted both the degree of BOLD activation during working memory and n-back performance. This phenomenon was present especially in the ventral portions of the LPFC bilaterally and was significantly greater in patients as compared with controls in the right ventral LPFC. Further post-hoc analyses revealed that the latter finding was also true in the MPFC, whereas in the parietal cortex patients showed a significantly more negative slope for the correlation as compared with controls. We speculate that during development subtle alterations in the distribution of thalamo-cortical pathways to their cortical targets, not necessarily manifest in anisotropy alterations, contribute to changes in the compartmentalization of dorsal and ventrolateral PFC function and to excessive VLPFC activation in schizophrenia (Tan et al, 2006). These changes may be compensatory in nature given the positive relationship to performance in both patients and controls. Moreover, also parietal and medial prefrontal nodes of the working memory network may be affected, although less severely.

From our data, it is impossible to say whether the thalamic abnormality in patients is a cause of, a consequence of, or a simultaneous occurrence with the LPFC abnormality. However, the fact that thalamocortical tracts form early in development suggests that this abnormality could already be present at birth or in the first years of life and would be consistent with the neurodevelopmental nature of schizophrenia. Indeed, there is evidence for altered interstitial prefrontal neurons, considered remnants of the subplate (Kostovic et al, 2011), in schizophrenia (Akbarian et al, 1993; Anderson et al, 1996; Eastwood and Harrison, 2003, 2005; Kirkpatrick et al, 2003; Yang et al, 2011), supporting an early pathological process. Furthermore, as the PFC completes maturation well after the thalamocortical fibers have reached the subplate (Kostovic and Judas, 2010), it is conceivable that thalamic neuronal activity might be a potential effector of PFC maturation and developing function via its connectivity (Kanold, 2009). An early presence of the abnormalities reported here is also supported by the lack of a correlation between CDR size and total percent connectivity with illness duration and other possible confounding variables such as age and chlorpromazine equivalents, although only studies in first-episode patients will be able to confirm this hypothesis.

This structure-function relationship supports the biological importance of our DTI findings when comparing patients and controls. We have linked DTI-derived measures of probabilistic connectivity with cortical physiology measured with fMRI and working memory performance, which is critically affected in the developmental pathophysiology of schizophrenia. Others have shown a relationship of FA and working memory performance in patients with 
schizophrenia (eg, Karlsgodt et al, 2008; Lim et al, 2006), and recent papers found correlations between working memory performance and anisotropy of the ALIC (Mamah et al, 2010) and of the left thalamus (Qiu et al, 2009) in schizophrenia, and mean diffusivity of the medial thalamus in healthy aging subjects (Charlton et al, 2010). Our findings also indicate that bulk thalamocortical connectivity is relevant to neurocognitive processes such as working memory.

Limitations of this study are the small number of subjects, inability to control for medication effects, and the fact that diagnostic groups were not well matched for years of education or premorbid IQ (although they did not differ in terms of parental socio-economic status, see Table 1), although this is commonly observed in naturalistic studies of schizophrenia. As the fMRI portion of the study was retrospective in nature, the demographics were not entirely representative of the full group, as in the case of sex distribution. Another limitation, especially in light of a recent report (Zhang et al, 2010) showing additive effects of smoking and schizophrenia on the FA of the ALIC, is the fact that $45 \%$ of our patients were current smokers compared with only one of our controls (5\%); however, this discrepancy was less marked when any history of smoking was taken into account (41\% of controls $v s 60 \%$ of patients). It should be noted, though that no group effect of FA was found in the ALIC in the current investigation. Finally, distortions of the diffusion-weighted images were not fully corrected by our post-processing scheme and this might have affected accurate registration with the T1weighted images. We are currently working on replicating these findings with a more rigorous post-processing scheme.

In conclusion, we have shown that putative measures of thalamocortical connectivity to LPFC were reduced in patients with schizophrenia and that there are functional implications of this reduction indicating the potential utility of DTI tractography measures in understanding white matter pathophysiology in schizophrenia.

\section{ACKNOWLEDGEMENTS}

This study was entirely funded by the NIMH IRP. The material in this paper was presented in abstract form at the annual meeting of the American College of Neuro-PsychoPharmacology in Boca Raton, FL (9-13 December 2007), at the meeting of the Society for Biological Psychiatry in Washington, DC (1-3 May 2008), and at the meeting of the International Schizophrenia Research Society in Venice, Italy (21-25 June 2008). This study utilized the highperformance computational capabilities of the Biowulf Linux cluster at the National Institutes of Health, Bethesda, MD (http://biowulf.nih.gov). We thank Fengyu Zhang (NIMH) for statistical advice, Tim Behrens and Saad Jbadi (Oxford) for guidance on probabilistic tractography, and Dharshan Chandramohan for help in the revision process.

\section{DISCLOSURE}

The authors declare no conflict of interest.

\section{REFERENCES}

Adriano F, Spoletini I, Caltagirone C, Spalletta G (2011). Updated meta-analyses reveal thalamus volume reduction in patients with first-episode and chronic schizophrenia. Schizophr Res 123: $1-14$.

Agarwal N, Rambaldelli G, Perlini C, Dusi N, Kitis O, Bellani M et al (2008). Microstructural thalamic changes in schizophrenia: a combined anatomic and diffusion weighted magnetic resonance imaging study. J Psychiatry Neurosci 33: 440-448.

Akbarian S, Vinuela A, Kim JJ, Potkin SG, Bunney Jr WE, Jones EG (1993). Distorted distribution of nicotinamide-adenine dinucleotide phosphate-diaphorase neurons in temporal lobe of schizophrenics implies anomalous cortical development. Arch Gen Psychiatry 50: 178-187.

Anderson SA, Volk DW, Lewis DA (1996). Increased density of microtubule associated protein 2-immunoreactive neurons in the prefrontal white matter of schizophrenic subjects. Schizophr Res 19: 111-119.

Andreasen NC, O'Leary DS, Cizadlo T, Arndt S, Rezai K, Ponto LL et al (1996). Schizophrenia and cognitive dysmetria: a positronemission tomography study of dysfunctional prefrontal-thalamic-cerebellar circuitry. Proc Natl Acad Sci USA 93: 9985-9990.

Andrews J, Wang L, Csernansky JG, Gado MH, Barch DM (2006). Abnormalities of thalamic activation and cognition in schizophrenia. Am J Psychiatry 163: 463-469.

Ashtari M, Cottone J, Ardekani BA, Cervellione K, Szeszko PR, Wu $J$ et al (2007). Disruption of white matter integrity in the inferior longitudinal fasciculus in adolescents with schizophrenia as revealed by fiber tractography. Arch Gen Psychiatry 64: 1270-1280.

Beasley CL, Dwork AJ, Rosoklija G, Mann JJ, Mancevski B, Jakovski $\mathrm{Z}$ et al (2009). Metabolic abnormalities in frontostriatal-thalamic white matter tracts in schizophrenia. Schizophr Res 109: 159-166.

Behrens TE, Berg HJ, Jbabdi S, Rushworth MF, Woolrich MW (2007). Probabilistic diffusion tractography with multiple fibre orientations: what can we gain? NeuroImage 34: 144-155.

Behrens TE, Johansen-Berg H, Woolrich MW, Smith SM, WheelerKingshott CA, Boulby PA et al (2003a). Non-invasive mapping of connections between human thalamus and cortex using diffusion imaging. Nat Neurosci 6: 750-757.

Behrens TE, Woolrich MW, Jenkinson M, Johansen-Berg H, Nunes RG, Clare $S$ et al (2003b). Characterization and propagation of uncertainty in diffusion-weighted MR imaging. Magn Reson Med 50: 1077-1088.

Buchsbaum MS, Friedman J, Buchsbaum BR, Chu KW, Hazlett EA, Newmark $\mathrm{R}$ et al (2006). Diffusion tensor imaging in schizophrenia. Biol Psychiatry 60: 1181-1187.

Byne W, Buchsbaum MS, Mattiace LA, Hazlett EA, Kemether E, Elhakem SL et al (2002). Postmortem assessment of thalamic nuclear volumes in subjects with schizophrenia. Am J Psychiatry 159: 59-65.

Byne W, Fernandes J, Haroutunian V, Huacon D, Kidkardnee S, Kim J et al (2007). Reduction of right medial pulvinar volume and neuron number in schizophrenia. Schizophr Res 90: 71-75.

Byne W, Hazlett EA, Buchsbaum MS, Kemether E (2009). The thalamus and schizophrenia: current status of research. Acta Neuropathol 117: 347-368.

Byne W, Kidkardnee S, Tatusov A, Yiannoulos G, Buchsbaum MS, Haroutunian V (2006). Schizophrenia-associated reduction of neuronal and oligodendrocyte numbers in the anterior principal thalamic nucleus. Schizophr Res 85: 245-253.

Byne W, Tatusov A, Yiannoulos G, Vong GS, Marcus S (2008). Effects of mental illness and aging in two thalamic nuclei. Schizophr Res 106: 172-181.

Callicott JH, Egan MF, Mattay VS, Bertolino A, Bone AD, Verchinksi B et al (2003a). Abnormal fMRI response of the 
dorsolateral prefrontal cortex in cognitively intact siblings of patients with schizophrenia. Am J Psychiatry 160: 709-719.

Callicott JH, Mattay VS, Verchinski BA, Marenco S, Egan MF, Weinberger DR (2003b). Complexity of prefrontal cortical dysfunction in schizophrenia: more than up or down. Am J Psychiatry 160: 2209-2215.

Charlton RA, Barrick TR, Lawes IN, Markus HS, Morris RG (2010). White matter pathways associated with working memory in normal aging. Cortex 46: 474-489.

Danos P, Schmidt A, Baumann B, Bernstein HG, Northoff G, Stauch $\mathrm{R}$ et al (2005). Volume and neuron number of the mediodorsal thalamic nucleus in schizophrenia: a replication study. Psychiatry Res 140: 281-289.

Desikan RS, Segonne F, Fischl B, Quinn BT, Dickerson BC, Blacker $\mathrm{D}$ et al (2006). An automated labeling system for subdividing the human cerebral cortex on MRI scans into gyral based regions of interest. NeuroImage 31: 968-980.

Dorph-Petersen KA, Pierri JN, Sun Z, Sampson AR, Lewis DA (2004). Stereological analysis of the mediodorsal thalamic nucleus in schizophrenia: volume, neuron number, and cell types. J Comp Neurol 472: 449-462.

Eastwood SL, Harrison PJ (2003). Interstitial white matter neurons express less reelin and are abnormally distributed in schizophrenia: towards an integration of molecular and morphologic aspects of the neurodevelopmental hypothesis. Mol Psychiatry 8: 769 .

Eastwood SL, Harrison PJ (2005). Interstitial white matter neuron density in the dorsolateral prefrontal cortex and parahippocampal gyrus in schizophrenia. Schizophr Res 79: 181-188.

Egan MF, Goldberg TE, Gscheidle T, Weirich M, Rawlings R, Hyde TM: et al (2001). Relative risk for cognitive impairments in siblings of patients with schizophrenia. Biol Psychiatry 50: 98-107.

Goldman AL, Pezawas L, Mattay VS, Fischl B, Verchinski BA, Zoltick B et al (2008). Heritability of brain morphology related to schizophrenia: a large-scale automated magnetic resonance imaging segmentation study. Biol Psychiatry 63: 475-483.

Harms MP, Wang L, Mamah D, Barch DM, Thompson PA, Csernansky JG (2007). Thalamic shape abnormalities in individuals with schizophrenia and their nonpsychotic siblings. J Neurosci 27: 13835-13842.

Hashimoto R, Mori T, Nemoto K, Moriguchi Y, Noguchi H, Nakabayashi $\mathrm{T}$ et al (2009). Abnormal microstructures of the basal ganglia in schizophrenia revealed by diffusion tensor imaging. World J Biol Psychiatry 10: 65-69.

Hazlett EA, Buchsbaum MS, Byne W, Wei TC, Spiegel-Cohen J, Geneve C et al (1999). Three-dimensional analysis with MRI and PET of the size, shape, and function of the thalamus in the schizophrenia spectrum. Am J Psychiatry 156: 1190-1199.

Hollingshead AB (1975). Four Factor Index of Social Status. Yale University: New Haven, CT.

Jenkinson M, Smith S (2001). A global optimisation method for robust affine registration of brain images. Med Image Anal 5: 143-156.

Johansen-Berg H, Behrens TE, Sillery E, Ciccarelli O, Thompson AJ, Smith SM et al (2005). Functional-anatomical validation and individual variation of diffusion tractography-based segmentation of the human thalamus. Cereb Cortex 15: 31-39.

Kanold PO (2009). Subplate neurons: crucial regulators of cortical development and plasticity. Front Neuroanat 3: 16.

Karlsgodt KH, van Erp TG, Poldrack RA, Bearden CE, Nuechterlein $\mathrm{KH}$, Cannon TD (2008). Diffusion tensor imaging of the superior longitudinal fasciculus and working memory in recent-onset schizophrenia. Biol Psychiatry 63: 512-518.

Kemether EM, Buchsbaum MS, Byne W, Hazlett EA, Haznedar M, Brickman AM et al (2003). Magnetic resonance imaging of mediodorsal, pulvinar, and centromedian nuclei of the thalamus in patients with schizophrenia. Arch Gen Psychiatry 60: 983-991.
Kessler RM, Woodward ND, Riccardi P, Li R, Ansari MS, Anderson $S$ et al (2009). Dopamine D2 receptor levels in striatum, thalamus, substantia nigra, limbic regions, and cortex in schizophrenic subjects. Biol Psychiatry 65: 1024-1031.

Kim DJ, Kim JJ, Park JY, Lee SY, Kim J, Kim IY et al (2008). Quantification of thalamocortical tracts in schizophrenia on probabilistic maps. Neuroreport 19: 399-403.

Kim JJ, Kim DJ, Kim TG, Seok JH, Chun JW, Oh MK et al (2007). Volumetric abnormalities in connectivity-based subregions of the thalamus in patients with chronic schizophrenia. Schizophr Res 97: 226-235.

Kirkpatrick B, Messias NC, Conley RR, Roberts RC (2003). Interstitial cells of the white matter in the dorsolateral prefrontal cortex in deficit and nondeficit schizophrenia. J Nerv Ment Dis 191: 563-567.

Kito S, Jung J, Kobayashi T, Koga Y (2009). Fiber tracking of white matter integrity connecting the mediodorsal nucleus of the thalamus and the prefrontal cortex in schizophrenia: a diffusion tensor imaging study. Eur Psychiatry 24: 269-274.

Klein JC, Rushworth MF, Behrens TE, Mackay CE, de Crespigny AJ, D'Arceuil H et al (2010). Topography of connections between human prefrontal cortex and mediodorsal thalamus studied with diffusion tractography. NeuroImage 51: 555-564.

Konick LC, Friedman L (2001). Meta-analysis of thalamic size in schizophrenia. Biol Psychiatry 49: 28-38.

Kostovic I, Judas M (2010). The development of the subplate and thalamocortical connections in the human foetal brain. Acta Paediatr 99: 1119-1127.

Kostovic I, Judas M, Sedmak G (2011). Developmental history of the subplate zone, subplate neurons and interstitial white matter neurons: relevance for schizophrenia. Int J Dev Neurosci 29: 193-205.

Kreczmanski P, Heinsen H, Mantua V, Woltersdorf F, Masson T, Ulfig $\mathrm{N}$ et al (2007). Volume, neuron density and total neuron number in five subcortical regions in schizophrenia. Brain 130(Part 3): 678-692.

Kubicki M, Park H, Westin CF, Nestor PG, Mulkern RV, Maier SE et al (2005). DTI and MTR abnormalities in schizophrenia: analysis of white matter integrity. NeuroImage 26: 1109-1118.

Kumari V, Fannon D, Ffytche DH, Raveendran V, Antonova E, Premkumar $\mathrm{P}$ et al (2010). Functional MRI of verbal selfmonitoring in schizophrenia: performance and illness-specific effects. Schizophr Bull 36: 740-755.

Kyriakopoulos M, Vyas NS, Barker GJ, Chitnis XA, Frangou S (2008). A diffusion tensor imaging study of white matter in early-onset schizophrenia. Biol Psychiatry 63: 519-523.

Lehrer DS, Christian BT, Mantil J, Murray AC, Buchsbaum BR, Oakes TR et al (2005). Thalamic and prefrontal FDG uptake in never medicated patients with schizophrenia. Am J Psychiatry 162: 931-938.

Lim KO, Ardekani BA, Nierenberg J, Butler PD, Javitt DC, Hoptman MJ (2006). Voxelwise correlational analyses of white matter integrity in multiple cognitive domains in schizophrenia. Am J Psychiatry 163: 2008-2010.

Lindenmayer JP, Bernstein-Hyman R, Grochowski S, Bark N (1995). Psychopathology of Schizophrenia: initial validation of a 5-factor model. Psychopathology 28: 22-31.

Mamah D, Conturo TE, Harms MP, Akbudak E, Wang L, McMichael AR et al (2010). Anterior thalamic radiation integrity in schizophrenia: a diffusion-tensor imaging study. Psychiatry Res 183: 144-150.

Meda SA, Jagannathan K, Gelernter J, Calhoun VD, Liu J, Stevens MC et al (2010). A pilot multivariate parallel ICA study to investigate differential linkage between neural networks and genetic profiles in schizophrenia. NeuroImage 53: 1007-1015.

Meyer-Lindenberg A, Weinberger DR (2006). Intermediate phenotypes and genetic mechanisms of psychiatric disorders. Nat Rev Neurosci 7: 818-827. 
Oh JS, Kubicki M, Rosenberger G, Bouix S, Levitt JJ, McCarley RW et al (2009). Thalamo-frontal white matter alterations in chronic schizophrenia: a quantitative diffusion tractography study. Hum Brain Mapp 30: 3812-3825.

Popken GJ, Bunney Jr WE, Potkin SG, Jones EG (2000). Subnucleus-specific loss of neurons in medial thalamus of schizophrenics. Proc Natl Acad Sci USA 97: 9276-9280.

Qiu A, Zhong J, Graham S, Chia MY, Sim K (2009). Combined analyses of thalamic volume, shape and white matter integrity in first-episode schizophrenia. NeuroImage 47: 1163-1171.

Rimol LM, Hartberg CB, Nesvag R, Fennema-Notestine C, Hagler Jr DJ, Pung CJ et al (2010). Cortical thickness and subcortical volumes in schizophrenia and bipolar disorder. Biol Psychiatry 68: $41-50$.

Rose SE, Chalk JB, Janke AL, Strudwick MW, Windus LC, Hannah $\mathrm{DE}$ et al (2006). Evidence of altered prefrontal-thalamic circuitry in schizophrenia: an optimized diffusion MRI study. NeuroImage 32: 16-22.

Schlosser R, Gesierich T, Kaufmann B, Vucurevic G, Stoeter P (2003). Altered effective connectivity in drug free schizophrenic patients. Neuroreport 14: 2233-2237.

Spoletini I, Cherubini A, Banfi G, Rubino IA, Peran P, Caltagirone $C$ et al (2011). Hippocampi, thalami, and accumbens microstructural damage in schizophrenia: a volumetry, diffusivity, and neuropsychological study. Schizophr Bull 37: 118-130.

Sprooten E, Lymer GK, Maniega SM, McKirdy J, Clayden JD, Bastin ME et al (2009). The relationship of anterior thalamic radiation integrity to psychosis risk associated neuregulin-1 variants. Mol Psychiatry 14: 237-238.

Sussmann JE, Lymer GK, McKirdy J, Moorhead TW, Munoz Maniega S, Job D et al (2009). White matter abnormalities in bipolar disorder and schizophrenia detected using diffusion tensor magnetic resonance imaging. Bipolar Disord 11: 11-18.

Szeszko PR, Ardekani BA, Ashtari M, Kumra S, Robinson DG, Sevy $S$ et al (2005). White matter abnormalities in first-episode schizophrenia or schizoaffective disorder: a diffusion tensor imaging study. Am J Psychiatry 162: 602-605.
Tan HY, Sust S, Buckholtz JW, Mattay VS, Meyer-Lindenberg A, Egan MF et al (2006). Dysfunctional prefrontal regional specialization and compensation in schizophrenia. $A m \mathrm{~J}$ Psychiatry 163: 1969-1977.

Traynor C, Heckemann RA, Hammers A, O'Muircheartaigh J, Crum WR, Barker GJ et al (2010). Reproducibility of thalamic segmentation based on probabilistic tractography. NeuroImage 52: 69-85.

Weinberger DR, Egan MF, Bertolino A, Callicott JH, Mattay VS, Lipska BK et al (2001). Prefrontal neurons and the genetics of schizophrenia. Biol Psychiatry 50: 825-844.

Welsh RC, Chen AC, Taylor SF (2010). Low-frequency BOLD fluctuations demonstrate altered thalamocortical connectivity in schizophrenia. Schizophr Bull 36: 713-722.

White T, Kendi AT, Lehericy S, Kendi M, Karatekin C, Guimaraes A et al (2007). Disruption of hippocampal connectivity in children and adolescents with schizophrenia-a voxel-based diffusion tensor imaging study. Schizophr Res 90: 302-307.

Woods SW (2003). Chlorpromazine equivalent doses for the newer antipsychotics. J Clin Psychiatry 64: 663-667.

Yang Y, Fung SJ, Rothwell A, Tianmei S, Weickert CS (2011). Increased interstitial white matter neuron density in the dorsolateral prefrontal cortex of people with schizophrenia. Biol Psychiatry 69: 63-70.

Young KA, Manaye KF, Liang C, Hicks PB, German DC (2000). Reduced number of mediodorsal and anterior thalamic neurons in schizophrenia. Biol Psychiatry 47: 944-953.

Zhang X, Stein EA, Hong LE (2010). Smoking and schizophrenia independently and additively reduce white matter integrity between striatum and frontal cortex. Biol Psychiatry 68: 674-677.

Zhou Y, Liang M, Jiang T, Tian L, Liu Y, Liu Z et al (2007). Functional dysconnectivity of the dorsolateral prefrontal cortex in first-episode schizophrenia using resting-state fMRI. Neurosci Lett 417: 297-302.

Zhou Y, Shu N, Liu Y, Song M, Hao Y, Liu H et al (2008). Altered resting-state functional connectivity and anatomical connectivity of hippocampus in schizophrenia. Schizophr Res 100: 120-132.

Supplementary Information accompanies the paper on the Neuropsychopharmacology website (http://www.nature.com/npp) 